\title{
Anaplasmataceae-Specific PCR for Diagnosis and Therapeutic Guidance for Symptomatic Neoehrlichiosis in Immunocompetent Host
}

\author{
Michael Schwameis, Julia Auer, \\ Dieter Mitteregger, Ingrid Simonitsch-Klupp, \\ Michael Ramharter, Heinz Burgmann, \\ Heimo Lagler
}

Candidatus Neoehrlichia is increasingly being recognized worldwide as a tickborne pathogen. We report a case of symptomatic neoehrlichiosis in an immunocompetent Austria resident who had recently returned from travel in Tanzania. The use of Anaplasmataceae-specific PCR to determine the duration of antimicrobial therapy seems reasonable to avert recrudescence.

$\mathrm{H}$ uman neoehrlichiosis is an infectious disease that primarily affects immunocompromised persons and persons with severe concurrent medical conditions (1-5). We describe symptomatic Candidatus Neoehrlichia infection in an otherwise healthy woman who had returned from a 28-day vacation in Tanzania, and we illustrate the applicability of Anaplasmataceae-specific PCR for diagnosis and therapeutic guidance.

\section{The Study}

In January 2013, a 30-year-old white woman with no relevant medical history was admitted to the Division of Infectious Diseases and Tropical Medicine, General Hospital of Vienna, in Vienna, Austria, because of a 3-week history of high fevers (up to $39.9^{\circ} \mathrm{C}$ ), chills, and night sweats accompanied by headache, muscle pain, and malaise. Four weeks before hospitalization, the woman had returned from a 28day vacation in Tanzania. She had not taken antimalarial prophylaxis drugs while in Tanzania; instead she carried atovaquone/proguanil tablets as a standby medication. The woman felt well during the entire stay in Tanzania, so she did not take the atovaquone/proguanil.

Spiking fevers began 5 days after her return to Vienna. She visited the outpatient clinic at the General Hospital of Vienna, where malaria, Dengue virus fever, and typhoid fever were ruled out as causative diseases. During her first days in Tanzania, the woman had voluntary skin contact with a prosimian, but she recalled no recent tick bites or exposures to animals. Blood samples were obtained; multiple cultures were negative. However, over the next 10 days,

Author affiliation: Medical University of Vienna, Vienna, Austria

DOI: http://dx.doi.org/10.3201/eid2202.141762 persistent fever and a deteriorating general condition led to hospitalization for further evaluation. Clinical data at admission and results of primary diagnostic tests are provided in the Table.

During the first days of hospitalization, the woman had fever (up to $39^{\circ} \mathrm{C}$ ) accompanied by general discomfort and headache. Giemsa-stained thick and thin blood smears showed no evidence of malaria. Likewise, serologic and PCR test results for Plasmodium spp. were negative.

On hospitalization day 5, a peripheral blood sample was tested by using a 16S rRNA gene-based eubacterial broad range PCR (SepsiTest; Molzym GmbH \& Co. KG, Bremen, Germany); results were positive. The amplification products $(300 \mathrm{bp})$ were sequenced (GenBank accession no. KT895260) and compared, using BLAST (http:// blast.ncbi.nlm.nih.gov/Blast.cgi), with known sequences in the National Center for Biotechnology Information (http:// www.ncbi.nlm.nih.gov/) database. The sequence showed 98\% (294/300 bp) homology with Candidatus Neoehrlichia lotoris (GenBank accession no. EF633744.1; only 1 database entry was available) and $97 \%$ (293/301 bp) homology with Candidatus Neoehrlichia mikurensis (GenBank accession no. KF155504.1; several database entries were available and showed a reproducible single base deletion at position 225). These findings were confirmed by Anaplasmataceae-specific 16S ribosomal RNA gene-based PCR. Primer pairs EHR16SD (5'-GGT ACC YAC AGA AGA AGT CC-3') and EHR16SR (5'-TAG CAC TCA TCG TTT ACA GC-3') were chosen to amplify a 345-bp fragment (6). The protocol was adjusted to that in the manual for High-Fidelity PCR enzyme mix (Thermo Scientific, Waltham, MA, USA) and to that of Brown et al. (7). Bidirectional sequencing of the 345-bp amplicon showed a sequence of $243 \mathrm{bp}$ corresponding to the cDNA strand (GenBank accession no. KT953340) and yielded similar results: $97 \%$ (235/243 bp) sequence homology was shared with Candidatus Neoehrlichia lotoris (GenBank accession no. EF633744.1), and 96\% (235/244 bp) sequence homology was shared with Candidatus Neoehrlichia mikurensis (GenBank accession no. JQ359046.1). Because the percentages of shared homologies were not sufficient to attribute the identified microbial agent to an official species, we tentatively named the agent Candidatus Neoehrlichia Tanzania. In addition, a microscopy review of Giemsa-stained blood smears obtained within the first days of admission 
Table. Clinical data at admission and primary diagnostic test results for a patient with Candidatus Neoehrlichia infection, Austria, 2013*

\begin{tabular}{|c|c|}
\hline Clinical variable & Finding/value \\
\hline Subjective symptoms & $\begin{array}{c}\text { Malaise, diffuse muscle pain, dull headache (without signs of meningism), and } \\
\text { tenderness in the left upper abdominal quadrant }\end{array}$ \\
\hline Tympanic temperature & $37.8^{\circ} \mathrm{C}$, while taking acetaminophen \\
\hline Heart auscultation & Systolic murmur (right sternal border), tachycardia (125 beats per minute) \\
\hline Condition of skin & No rash or signs of cutaneous exposure to arthropods \\
\hline \multicolumn{2}{|l|}{ Laboratory testing $\dagger$} \\
\hline C-reactive protein & $5 \mathrm{mg} / \mathrm{dL}(<0.5)$ \\
\hline Procalcitonin & $0.14 \mathrm{ng} / \mathrm{mL}(<0.5)$ \\
\hline Leukocyte count & $3.9 \times 10^{9} / L(4-10)$ \\
\hline Neutrophils & $53 \%(50-75)$ \\
\hline Lymphocytes & $27 \%(25-40)$ \\
\hline Monocytes & $16 \%(0-12)$ \\
\hline Fibrinogen & 480 mg/dL (180-390) \\
\hline Serum amyloid $A$ & $164 \mathrm{mg} / \mathrm{L}(<5)$ \\
\hline$\gamma$-globulins & $26.2 \%(11.1-18.8)$ \\
\hline Erythrocyte sedimentation rate & $70 \mathrm{~mm} / \mathrm{h}(<15)$ \\
\hline Platelet count & $121 \times 10^{9} / L(150-350)$ \\
\hline Hemoglobin & $9 \mathrm{~g} / \mathrm{dL}(12-16)$ \\
\hline Chest radiography & No consolidations, no opacities \\
\hline Abdominal ultrasonography & Splenomegaly of $15.5 \times 6.7 \mathrm{~cm}$ \\
\hline Transesophageal echocardiography & Normal cardiac function and valves, no evidence of vegetations \\
\hline Cranial computed tomography & Parasagittal meningioma, otherwise normal \\
\hline Ophthalmologic examination & Bilateral papilloedema \\
\hline Cerebrospinal fluid & $\begin{array}{c}\text { Clear and colorless; absolute cell count } 4 / \mu \mathrm{L} \text { protein, glucose, and lactate levels } \\
\text { within reference range }\end{array}$ \\
\hline Abdominal ultrasonography & Splenomegaly, $15.5 \times 6.7 \mathrm{~cm}$ \\
\hline Urinary dip stick and urinary cultures & No growth \\
\hline Blood cultures & No growth \\
\hline \multicolumn{2}{|l|}{ Serologic testing } \\
\hline HIV & Negative \\
\hline Hepatitis B and C viruses & Negative \\
\hline Epstein-Barr virus & Negative \\
\hline Cytomegalovirus & Negative \\
\hline Mycoplasma spp. & Negative \\
\hline Adenovirus & Negative \\
\hline Enterovirus & Negative \\
\hline Coxsackievirus & Negative \\
\hline Influenza $A, B$, and $C$ viruses & Negative \\
\hline Parainfluenza virus & Negative \\
\hline Anaplasma spp. & Negative \\
\hline Rickettsia spp. & Negative \\
\hline Tuberculous mycobacteria & Negative \\
\hline Plasmodium spp. & Negative \\
\hline Syphilis (VDRL, TPPA) & Negative \\
\hline \multicolumn{2}{|l|}{ PCR testing } \\
\hline Leishmania spp. & Negative \\
\hline Trypanosoma spp. & Negative \\
\hline Plasmodium spp. & Negative \\
\hline \multicolumn{2}{|l|}{ Giemsa-stained thin and thick blood smears } \\
\hline Plasmodium spp. & Negative \\
\hline
\end{tabular}

showed structures possibly equivalent to microbial pathogens within leukocytes (Figure 1).

Antimicrobial treatment with oral doxycycline (300 mg per day) was subsequently initiated, resulting in improvement in the patient's overall condition within 2 days and in a continuous decrease of all inflammation markers, normalization of platelet counts, and abatement of fever (Figure 2). However, serum Neoehrlichia DNA remained detectable at high levels. To provide the optimal duration of antibiotic treatment, we performed daily Anaplasmataceae-specific 16S PCR measurements of blood samples. Over the next 10 days of therapy, the DNA signal intensity continuously diminished. Doxycycline was stopped 1 day after disappearance of Neoehrlichia serum DNA.

In contrast with patients in previously published reports of human neoehrlichiosis, the patient described in our report was a healthy young woman without concurrent medical conditions. She had signs and symptoms of disease 


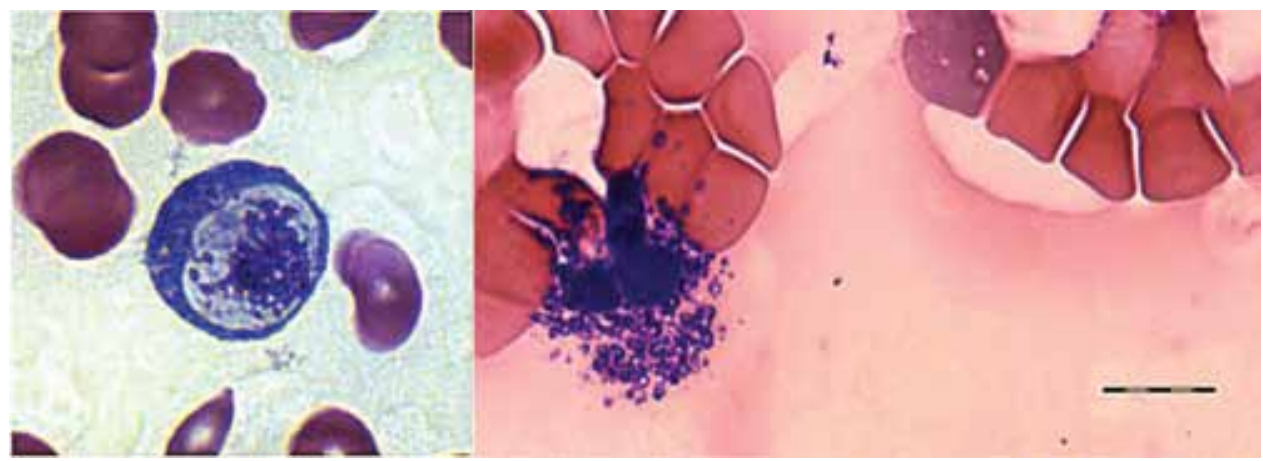

Figure 1. Giemsa-stained blood smear from an immunocompetent patient with Candidatus Neoehrlichia infection, Austria, 2013.

The blood smear shows possible microbial pathogens within leukocytes. Scale bar indicates $10 \mu \mathrm{m}$.

for 4 weeks without any symptomatic improvement before therapy was initiated. Treatment led to a rapid clinical response and rapid clearance of serum Neoehrlichia DNA, which may be attributable to her otherwise good medical condition but may also reflect high antimicrobial efficiency of the high-dose therapeutic regimen applied.

Because symptomatic neoehrlichiosis usually occurs in patients with immunosuppression, we examined the patient for an underlying malignancy or autoimmune disorder. These conditions were largely ruled out by negative test results for HIV and mycobacteria and by a normal finding on 18F-FDG-PET/CT (18F-fluordeoxyglucosepositron emission tomography/computed tomography) examination (except for enhanced splenic FDG uptake). The patient had moderate disease with nonspecific symptoms partly resembling those of human anaplasmosis. The splenomegaly was attributed to polyclonal B cell activity (indicated by hypergammaglobulinemia), but it could also have resulted from direct infection of splenic sinusoidal cells, as found in Neoehrlichia-infected Wistar rats (8). However, spleen size decreased over the course of antimicrobial treatment and reached a normal diameter by a 3-week follow-up examination.

No evidence exists regarding the exact incubation period of human neoehrlichiosis, but it probably approximates that of human granulocytic anaplasmosis, suggesting that the patient in our study acquired neoehrlichiosis in Tanzania. Nonetheless, several tickborne diseases are highly endemic in Austria. Glatz et al. (9) recently reported a $4.2 \%$ prevalence of Candidatus Neoehrlichia in Ixodes ricinus ticks in Austria. However, in the 5-day period between returning home from Tanzania and fever onset, the patient in our study had stayed in the urban area of Vienna; thus, the possibility that she may have been

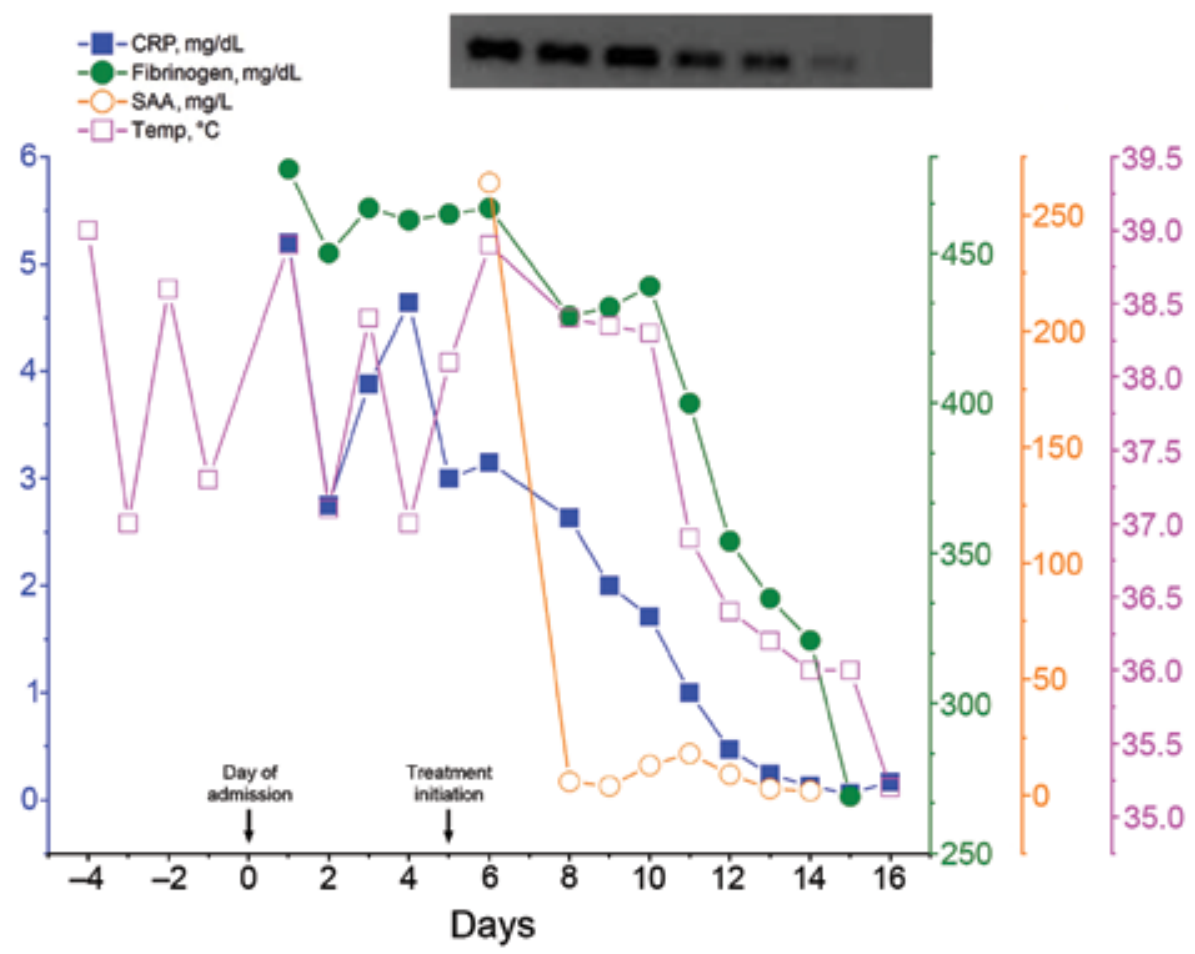

Figure 2. Body temperature and markers of inflammation over the course of hospitalization for a patient with Candidatus Neoehrlichia infection, Austria, 2013. Day 0 indicates time of admission. Antimicrobial therapy with doxycycline (300 mg per day) was begun on day 5 and led to a rapid resolution of clinical symptoms and a progressive decrease of all inflammatory parameters. Daily Anaplasmataceae-specific PCR measurements guided therapy, which was safely stopped 1 day after disappearance of serum Candidatus Neoehrlichia DNA. Upper right shows $1.5 \%$ agarose gel electrophoresis analysis. The intensity of the 345-bp DNA band amplified from blood samples progressively decreased over the course of treatment. CRP, C-reactive protein; SAA, serum amyloid $A$; Temp, tympanic temperature. 
exposed to ticks in Austria is limited but not excluded. Furthermore, the patient returned to Vienna at the height of winter, making the possible transmission of Candidatus Neoehrlichia by a domestic tick even less plausible. On the other hand, no epidemiologic data are available on the prevalence of Candidatus Neoehrlichia in ticks in Tanzania, but Candidatus Neoehrlichia mikurensis was recently found in ticks of 2 species collected in Nigeria (10). Thus, the presence of Candidatus Neoehrlichia in ticks in Tanzania and the risk for transmission from ticks to humans seem conceivable. Because a $16 \mathrm{~S}$ rDNA sequence difference of $>2 \%$ is arbitrarily considered as indicative for delineation at the species level, it seems possible that a new Candidatus Neoehrlichia agent was detected in the patient in our study.

\section{Conclusions}

This case demonstrates that Candidatus Neoehrlichia can affect healthy persons who have no underlying hematologic or autoimmune disorders. Neoehrlichiosis should be considered in the differential diagnosis for patients with appropriate symptoms, independent of concurrent conditions and immune status. As long as no evidence-based recommendations regarding treatment of human neoehrlichiosis exist, it seems reasonable to use Anaplasmataceae-specific PCR to monitor treatment response and determine the duration of antimicrobial therapy to avert recrudescence.

\section{Acknowledgments}

We thank the patient in our study for giving consent to publish the data, Wolfgang Barousch for providing assistance with GenBank, and Albert Lalremruata for performing the Plasmodium spp. PCR.

Dr. Schwameis is an internal medicine resident at the Department of Clinical Pharmacology, Medical University of Vienna. His research interests include staphylococcal blood stream infections and infection-associated coagulopathy.

\section{References}

1. Welinder-Olsson C, Kjellin E, Vaht K, Jacobsson S, Wenneras C. First case of human "Candidatus Neoehrlichia mikurensis" infection in a febrile patient with chronic lymphocytic leukemia. J Clin Microbiol. 2010;48:1956-9. http://dx.doi.org/10.1128/ JCM.02423-09
2. Pekova S, Vydra J, Kabickova H, Frankova S, Haugvicova R, Mazal O, et al. Candidatus Neoehrlichia mikurensis infection identified in 2 hematooncologic patients: benefit of molecular techniques for rare pathogen detection. Diagn Microbiol Infect Dis. 2011;69:266-70. http://dx.doi.org/10.1016/ j.diagmicrobio.2010.10.004

3. von Loewenich FD, Geissdorfer W, Disque C, Matten J, Schett G, Sakka SG, et al. Detection of "Candidatus Neoehrlichia mikurensis" in two patients with severe febrile illnesses: evidence for a European sequence variant. J Clin Microbiol. 2010;48: 2630-5. http://dx.doi.org/10.1128/JCM.00588-10

4. Grankvist A, Andersson PO, Mattsson M, Sender M, Vaht K, Hoper L, et al. Infections with the tick-borne bacterium "Candidatus Neoehrlichia mikurensis" mimic noninfectious conditions in patients with B cell malignancies or autoimmune diseases. Clin Infect Dis. 2014;58:1716-22. http://dx.doi.org/ 10.1093/cid/ciu189

5. Fehr JS, Bloemberg GV, Ritter C, Hombach M, Luscher TF, Weber R, et al. Septicemia caused by tick-borne bacterial pathogen Candidatus Neoehrlichia mikurensis. Emerg Infect Dis. 2010;16:1127-9. http://dx.doi.org/10.3201/eid1607.091907

6. Inokuma H, Raoult D, Brouqui P. Detection of Ehrlichia platys DNA in brown dog ticks (Rhipicephalus sanguineus) in Okinawa Island, Japan. J Clin Microbiol. 2000;38:4219-21.

7. Brown GK, Martin AR, Roberts TK, Aitken RJ. Detection of Ehrlichia platys in dogs in Australia. Aust Vet J. 2001;79:554-8. http://dx.doi.org/10.1111/j.1751-0813.2001.tb10747.x

8. Kawahara M, Rikihisa Y, Isogai E, Takahashi M, Misumi H, Suto C, et al. Ultrastructure and phylogenetic analysis of 'Candidatus Neoehrlichia mikurensis' in the family Anaplasmataceae, isolated from wild rats and found in Ixodes ovatus ticks. Int J Syst Evol Microbiol. 2004;54:1837-43. http://dx.doi.org/10.1099/ijs.0.63260-0

9. Glatz M, Mullegger RR, Maurer F, Fingerle V, Achermann Y, Wilske B, et al. Detection of Candidatus Neoehrlichia mikurensis, Borrelia burgdorferi sensu lato genospecies and Anaplasma phagocytophilum in a tick population from Austria. Ticks Tick Borne Dis. 2014;5:139-44. http://dx.doi.org/10.1016/ j.ttbdis.2013.10.006

10. Kamani J, Baneth G, Mumcuoglu KY, Waziri NE, Eyal O, Guthmann Y, et al. Molecular detection and characterization of tick-borne pathogens in dogs and ticks from Nigeria. PLoS Negl Trop Dis. 2013;7:e2108. http://dx.doi.org/10.1371/ journal.pntd.0002108

Address for correspondence: Heimo Lagler, Department of Medicine I, Division of Infectious Diseases and Tropical Medicine, Medical University of Vienna, Waehringer Guertel 18-20, A-1090 Vienna, Austria; email: heimo.lagler@meduniwien.ac.at; Michael Schwameis, Department of Clinical Pharmacology, Medical University of Vienna, Waehringer Guertel 18-20, A-1090 Vienna, Austria; email: michael. schwameis@meduniwien.ac.at

\section{Letters}

Letters commenting on recent articles as well as letters reporting cases, outbreaks, or original research are welcome. Letters commenting on articles should contain no more than 300 words and 5 references; they are more likely to be published if submitted within 4 weeks of the original article's publication.
Letters reporting cases, outbreaks, or original research should contain no more than 800 words and 10 references. They may have 1 Figure or Table and should not be divided into sections. All letters should contain material not previously published and include a word count. 